\title{
Participación local. Más allá de la democracia asociativa $^{1}$
}

\author{
Joan Font e Ismael Blanco \\ Universitat Autònoma de Barcelona
}

\begin{abstract}
Sumario: 1. INTRODUCCIÓN. 2. ¿POR QUÉ IR MÁS ALLÁ DE LOS CONSEJOS CONSULTIVOS MUNICIPALES? 3. ¿CÓMO IR MÁS ALLÁ DE LOS CONSEJOS CONSULTIVOS MUNICIPALES? 3.1. Tipos de mecanismos. 3.2. Representatividad de los participantes. 3.3. El impacto en las políticas. 3.4. Los efectos educativos. 4. CONCLUSIONES. REFERENCIAS.
\end{abstract}

\section{INTRODUCCIÓN}

Planes estratégicos, Agendas 21, Jurados ciudadanos, Presupuestos participativos o Consejos infantiles son algunas de las etiquetas referentes a procesos que incorporan cierto componente de participación ciudadana que se han ido desarrollando a lo largo de los últimos años. Analizar qué significan y qué suponen respecto a los restantes mecanismos formalizados de participación que funcionan hace algunas décadas en las ciudades europeas es el principal objetivo que pretendemos exponer.

En este trabajo vamos a obviar la justificación de por qué resulta necesario abrir más espacios a la participación ciudadana en la elaboración de políticas públicas, tema que hemos desarrollado en otras partes (FONT y Blanco, 2001; Subirats, 2001) ${ }^{2}$. Partiendo por tanto del supuesto de que dicha participación resulta necesaria, el argumento que aquí se desarrolla hace referencia al cómo y al quién de estos procesos participativos ${ }^{3}$. Con-

\footnotetext{
${ }^{1}$ Versiones anteriores de este artículo han sido presentadas en el Seminari Sobre Nova Política (CCCB), en el Congreso de la Asociación Española de Ciencia Política (La Laguna, setiembre 2001) y en el congreso Developments in public participation and innovations in community governance (Bellaterra, junio 2001). Queremos agradecer a los participantes en estos diversos foros sus comentarios a este trabajo, que se ha visto beneficiado por todas estas aportaciones.
}

${ }^{2}$ Este trabajo debe entenderse como fruto de un trabajo de equipo por parte de las personas que trabajamos sobre participación ciudadana en el Equip d'Anàlisi Política. Concretamente, la primera parte se basa en argumentos similares a los que hemos desarrollado en FonT y BlanCo (2001), mientras que el material de la segunda parte procede de un trabajo colectivo (SuBIRATs et al., 2000).

3 También obviaremos otro debate fundamental para una plena comprensión de estos mecanismos, ¿qué lleva a sus impulsores a ponerlos en marcha? Probablemente estas motivaciones son muy diversas y en muchos casos tienen un fuerte componente instrumental (NAVARRO, 1999), pero consideramos que los argumentos que se desarrollan a continuación son válidos cualquiera que sea la 
REAL-2002, núm. 290. FONT, JOAN y BLANCO, ISMAEL. PARTICIPACIÓN LOCAL. MÁS ALLÁ ...

REAL 290 (SEPTIEMBRE-DICIEMBRE, 2002)

cretamente, queremos analizar una serie de mecanismos y experiencias que han tenido un gran desarrollo a lo largo de los últimos años y que tienen en común el hecho de ir más allá de los instrumentos de democracia asociativa de los que se han dotado buena parte de los ayuntamientos españoles y europeos desde hace años (NAVARRO, 1999). Es decir, aquí estudiaremos todos aquellos mecanismos de participación que supongan alguna innovación respecto a los consejos consultivos municipales, de base sectorial o territorial, cuya presencia se ha generalizado en los municipios grandes y medianos de nuestro entorno ${ }^{4}$.

Para ello, estructuraremos el trabajo en dos grandes apartados. En el primero trataremos de justificar por qué resulta interesante la puesta en marcha de este tipo de instrumentos de participación que, generalmente, consisten en ampliar de algún modo los sujetos participativos más allá de los grupos organizados, dando voz de alguna manera a la ciudadanía a título individual. Utilizando como marco analítico los mismos grandes argumentos que expondremos para justificar las ventajas de estos mecanismos de participación, analizaremos después el funcionamiento de cerca de 50 experiencias de este tipo que se han desarrollado a lo largo de los últimos años en ayuntamientos catalanes.

\section{2. ¿POR QUÉ IR MÁS ALLÁ DE LOS CONSEJOS CONSULTIVOS MUNICIPALES?}

Los trabajos analíticos en profundidad sobre el funcionamiento de los Consejos consultivos municipales son aún muy escasos, aunque empieza a existir un pequeño volumen de obras al respecto. Sin duda, sería necesario contar con un aporte mucho más amplio y plural para poder hacer un diagnóstico fiable de hasta qué punto éstos han sido instrumentos útiles y con qué fines. Entre otras cosas, porque si una conclusión parece destacar por encima de las demás en la literatura publicada es la que constata su extrema diversidad de funcionamiento real, por mucho que en sus aspectos más formales (composición, objetivos declarados o normas internas de

motivación original, siempre que el proceso participativo suponga algún tipo de resultados más allá de los publicitarios.

4 Utilizaremos como sinónimos el concepto de democracia asociativa y su plasmación institucional en Consejos consultivos municipales, aunque se trate de palabras que se mueven en dos planos muy distintos (teórico en un caso y empírico en el otro) y que por tanto en muchos casos no coincidan plenamente. Para el concepto de democracia asociativa, ver por ejemplo CoHEN y RoGERS (1995). 
REAL-2002, núm. 290. FONT, JOAN y BLANCO, ISMAEL. PARTICIPACIÓN LOCAL. MÁS ALLÁ ...

PARTICIPACIÓN LOCAL. MÁS ALLÁ DE LA DEMOCRACIA ASOCIATIVA

funcionamiento) muchos de ellos sigan patrones similares. Por ejemplo, la gran mayoría están formados sobre todo por representantes del municipio y de asociaciones, cuentan con plenario, comisión permanente y grupos de trabajo y ejercen un rol fundamentalmente consultivo. Pero en la práctica, la representación de las asociaciones puede ser extremadamente amplia y fragmentada o tendente a un modelo casi-oligopólico, los grupos de trabajo pueden ejercer un rol marginal o ser el verdadero espacio de reflexión y negociación del Consejo y éste puede realmente servir para mantener informadas a las asociaciones de las actuaciones municipales o convertirse en un verdadero espacio de diálogo, en el que se hacen propuestas elaboradas que terminan convertidas en ordenanzas o presupuestos municipales.

Pero, en cualquier caso, sí parece claro que incluso en aquellos Consejos que han funcionado relativamente bien y han resultado útiles, existen ciertas críticas generalizadas a su funcionamiento o algunas limitaciones que podría resultar necesario superar. Tratando de combinar las preocupaciones de asociaciones, de técnicos y políticos municipales y aquellas que se derivan de un análisis más académico de su funcionamiento, podríamos detectar tres grandes criterios, en los que el rendimiento de los consejos parece mejorable: la representatividad de los participantes, su capacidad de influir en las políticas públicas municipales y su potencial como instrumento de aprendizaje democrático ${ }^{5}$.

Una de las funciones principales de los procesos electorales en el marco de las democracias representativas es garantizar la representación de los intereses de los distintos ciudadanos y grupos sociales en los procesos de gobierno. La introducción de fórmulas de participación ciudadana más allá de las elecciones debería no sólo preservar sino potenciar la capacidad de representación de ese conjunto de intereses en las decisiones públicas ${ }^{6}$. No obstante, una vez abrimos nuevos espacios a la participación, la cuestión de la representación no queda resuelta automáticamente. Por una parte, los mecanismos de participación pueden verse afectados por sesgos participativos intensos derivados, entre otras razones, de la exigencia de unos recursos a los participantes que están desigualmente distribuidos entre la población. En este sentido, uno de los riesgos principales de las

\footnotetext{
${ }^{5}$ Para una profundización en los argumentos sobre por qué estos tres objetivos resultan deseables en un proceso de participación ver FONT y BLANCO (1991).

${ }^{6}$ Utilizamos el concepto de representatividad en su acepción más sociológica, es decir, que los participantes constituyan una buena fotografía a pequeña escala del conjunto de características e intereses de la población a la que se pretende escuchar. Para la discusión del concepto de representatividad aplicado a la participación ciudadana ver SAWARD (2000).
} 
REAL-2002, núm. 290. FONT, JOAN y BLANCO, ISMAEL. PARTICIPACIÓN LOCAL. MÁS ALLÁ ...

REAL 290 (SEPTIEMBRE-DICIEMBRE, 2002)

fórmulas de participación no electoral será premiar las opiniones y los intereses de los ciudadanos o los grupos con más recursos para participar, o dicho de otra forma, que la participación no sea representativa del conjunto de intereses y demandas presentes en la población. Por otra parte, no está claro qué tipo de ciudadanos nos interesa integrar en los procesos participativos, es decir, cuál es la voz o las voces que deben ser escuchadas en los procesos de participación ciudadana. En los diversos instrumentos participativos que analizaremos hay implícitos modelos de participación muy distintos, que divergen en cuanto a los puntos de vista e intereses a los que dan cabida.

El modelo de participación predominante ha potenciado la participación de los grupos organizados, en detrimento de la capacidad de los ciudadanos no organizados de incidir en los procesos de gobierno. Las razones son diversas. Por una parte, los gobiernos locales han concebido las asociaciones como interlocutores válidos de los intereses, las necesidades y las demandas presentes en la ciudadanía. Por otra parte, les resulta más fácil dialogar con grupos organizados que con ciudadanos que sólo pueden representarse a sí mismos, y que en la mayor parte de los casos están muy desinformados. Pero, además, los gobiernos han entendido que el potencial disruptivo más fuerte proviene de los colectivos organizados, con intereses y expectativas fuertes sobre la acción de gobierno, y con capacidad para influir y movilizar la opinión pública, por lo que éstos son los primeros en ser escuchados.

Desde finales de los años 80, el modelo de participación de base asociativa ha mostrado limitaciones importantes, buena parte de las cuales se refieren al presupuesto de la representatividad. En primer lugar, el desarrollo práctico de este modelo de participación permite dudar en muchos casos de la representatividad de los grupos participantes en relación con el conjunto del tejido asociativo. En segundo lugar, algunos de los ejemplos analizados demuestran que los asistentes a los órganos de participación no siempre se pueden presentar como representativos de las propias entidades de las que forman parte. Finalmente, y quizás lo más importante, las entidades tienen verdaderos problemas para garantizar su capacidad de representación de los intereses presentes en la población en un contexto de escasa participación asociativa.

En general, no todas las entidades que forman parte del tejido asociativo de un territorio o de un área temática logran estar representadas en los órganos y los procesos participativos. Un tipo determinado de asociaciones tiende a verse más favorecido que otro en estos espacios participativos, la composición de los cuales se ve condicionada por los mismos intereses 
REAL-2002, núm. 290. FONT, JOAN y BLANCO, ISMAEL. PARTICIPACIÓN LOCAL. MÁS ALLÁ ...

PARTICIPACIÓN LOCAL. MÁS ALLÁ DE LA DEMOCRACIA ASOCIATIVA

políticos de los gobernantes. Así, a lo largo de los años 80 los gobiernos municipales han tendido a favorecer la participación de los grupos más próximos ideológicamente, y con mayor capacidad de movilización social, mientras que la tendencia en estos últimos años es premiar a aquellas entidades con más capacidad de gestión y que muestren acuerdo con el gobierno en la forma de gestionar los servicios (NAVARRO, 1999). El caso de Barcelona ejemplifica la existencia de este nuevo tipo de sesgo, tanto en los consejos sectoriales como en los órganos de participación por distritos. En estos órganos se han visto favorecidas las asociaciones mejor adaptadas a la colaboración con las administraciones en la prestación de servicios públicos (BRUGUÉ y otros, 2001).

También son abundantes los casos en que la capacidad de los participantes en los órganos de participación para representar a sus propias entidades es dudosa. El caso de Barcelona es de nuevo un buen ejemplo del problema del envejecimiento y la falta de renovación de los participantes. En parte, este problema de representatividad puede deberse a una falta de interés de las entidades en las actividades de estos órganos, pero sobre todo, a las dificultades para encontrar personas disponibles y preparadas y a la insuficiente democracia interna en las mismas asociaciones. Así pues, es habitual encontrarnos con asociaciones que no prevén ningún tipo de espacio de comunicación e interacción entre los supuestos representantes y los miembros de la organización, con lo cual los participantes adquieren plena autonomía respecto al grupo social al que pretenden representar.

Finalmente, otro problema relevante de este modelo de participación es la debilidad del mismo tejido asociativo, un problema que afecta con desigual intensidad a la mayoría de las democracias occidentales. En un contexto de escasa participación asociativa, se puede dudar de la capacidad de los grupos que forman parte de este tejido asociativo para representar la voz del conjunto de los ciudadanos, un problema que toma especial relieve en el marco de un modelo de participación que ha dejado de lado a los no organizados ${ }^{7}$.

En cualquier caso, si pensamos que muchas de estas limitaciones del mundo asociativo han sido favorecidas por un modelo participativo que genera frustración y burocratización, quizás deberíamos concluir que los problemas de representatividad podrían ser superados con la recuperación de un modelo de participación que enfatice el rol crítico y de control

\footnotetext{
7 Básicamente estamos recuperando el argumento con el que OLson empieza su obra: la mayor parte de los intereses colectivos no llega a expresarse en ningún tipo de organización, debido a los problemas de la acción colectiva que él trata de explicar en su trabajo.
} 
democrático de las asociaciones, a la vez que con una profunda renovación de las mismas, que debe ser impulsada desde dentro de las entidades, y que puede ser apoyado y estimulado por las administraciones.

Ligado a los déficit de la participación de base asociativa, en los últimos años se ha abierto una tendencia a buscar nuevas fórmulas de participación que confieran protagonismo a los ciudadanos no organizados, ya sea a través de un modelo de participación mixta o bien de base exclusivamente personal. El caso de la planificación estratégica sería un ejemplo ilustrativo de esta tendencia. Mientras que en sus primeras versiones se contó con la participación de las empresas ligadas al desarrollo socioeconómico del territorio, más tarde se empezó a favorecer la participación del conjunto de entidades del tejido asociativo y en los últimos años, en la mayoría de procesos de planificación estratégica, se abre la participación a los ciudadanos no organizados. En los mecanismos participativos que analizaremos aquí no vamos a encontrar necesariamente mejores resultados en términos de representatividad que en los Consejos consultivos municipales, pero sí es cierto que muchos de ellos parten de esta voluntad de superar los déficit de representatividad descritos, por medio de incorporar instrumentos de participación para los ciudadanos a título individual.

Si al hablar sobre los déficit de los Consejos consultivos los técnicos y políticos locales apuntan a menudo a los problemas de representatividad, el mundo asociativo señala reiteradamante las dificultades para lograr un impacto significativo de sus debates y decisiones en las políticas públicas locales. En aquellos Consejos que han tenido un funcionamiento correcto existe, cuando menos, un efecto en la detección compartida de necesidades y el establecimiento de espacios de diálogo, junto a la puesta en marcha de determinados mecanismos de accountability, mediante los cuales los participantes han podido tomar consciencia de hasta qué punto el gobierno local tomaba o no en consideración sus recomendaciones. Pero estos casos son más la excepción que la regla y en general la relativa frustración que se detecta se debe a la falta de compensación entre un gran esfuerzo que las asociaciones necesitan hacer para mantenerse activas en los consejos y los escasos resultados reales que éstos llegan a producir.

Sin duda podría objetarse que los limitados resultados de estos consejos en el terreno de su influencia en las políticas se deben a que ésta nunca fue su función, pues siempre se han concebido como espacios para informar al movimiento asociativo o, en el mejor de los casos, para consultar y discutir con éste las políticas previstas. Asimismo, desde una institución de gobierno que se basa en una lógica representativa, la incorporación de funciones que vayan más allá del ámbito estrictamente consultivo puede 
REAL-2002, núm. 290. FONT, JOAN y BLANCO, ISMAEL. PARTICIPACIÓN LOCAL. MÁS ALLÁ ...

PARTICIPACIÓN LOCAL. MÁS ALLÁ DE LA DEMOCRACIA ASOCIATIVA

entrar en aparente contradicción con el mandato dado a los cargos electos y su necesidad de responder ante los ciudadanos como responsables de las actuaciones desarrolladas en la siguiente convocatoria electoral.

Sin embargo, existen argumentos de muy diversa índole para pensar que la puesta en marcha de instrumentos participativos que no dispongan de cierta capacidad real de influir en el proceso decisional es un ejercicio absurdo y que sólo aboca a la frustración. Quizás el motivo principal es la dificultad de convencer a los propios participantes de asumir un rol activo si no está nada claro para qué va a servir dicha participación. No se trata de tener que dar garantías a priori de que van a seguirse todas sus recomendaciones, pero sí de que va a tomarse en serio su trabajo y de que no está participando en un ejercicio puramente ritual. Al menos, algunos análisis de las motivaciones de los participantes apuntan en la misma línea subrayada por algunos de los críticos del trabajo de Olson, en el sentido de que la voluntad de influir en las políticas es el principal elemento motivador para participar, por mucho que esto entre en contradicción aparente con la lógica del free-rider (SChlozMAn, Verba y BRAdy, 199).

Un tema clave es el encaje entre estos mecanismos y un proceso de toma de decisiones, donde la última palabra va a corresponder siempre a los representantes electos (Delap, 2001). Desde esta perspectiva, si el proceso de participación forma parte del proyecto político que los representantes electos han explicado a sus electores, si en ese proceso participativo los representantes juegan también un rol que permita que se escuche su voz con el protagonismo suficiente, si las reglas y responsabilidades de cada uno quedan claramente delimitadas desde un principio y si existen los mecanismos de rendición de cuentas para que los representantes puedan explicar a posteriori sus decisiones a los participantes, es posible que participación y representación pueden convertirse en procesos más complementarios que contradictorios. La responsabilidad de los representantes puede no sólo no estar amenazada, sino verse incluso reforzada. Por ejemplo, el ciclo de los presupuestos participativos empieza con el alcalde rindiendo cuentas de la ejecución del presupuesto del año anterior (ABERs, 2000), la primera reunión anual del Consejo Municipal de Bienestar Social (CMBS) de Barcelona utiliza exactamente el mismo procedimiento y algunas experiencias de jurados como el de Camden (Londres) incorporan una sesión a posteriori en la que los políticos explican las propuestas que van a derivarse de sus recomendaciones a los que habían sido sus integrantes. Otro ejemplo en esta línea fue el referéndum telefónico organizado por los diputados del Reform Party canadiense, que se mostraron dispuestos a vincular al mismo su votación parlamentaria si dicho referéndum 
conseguía una participación del $70 \%$ de los consultados, lo cual ocurrió en algunos de los distritos.

En cualquier caso, la traducción de la participación en políticas va a ser muy desigual dependiendo de dos factores. En primer lugar, el tipo de instrumentos utilizados va a ser importante, pues mientras presupuestos participativos o jurados ciudadanos están muy orientados a tomar decisiones, los Consejos municipales o las Agendas 21 también pueden hacerlo pero tienen un formato que prioriza el diálogo y el intercambio por encima de la capacidad decisional. Pero, en segundo lugar, la voluntad política de hacer un uso u otro de los mecanismos va a ser decisiva y va a provocar que un consejo municipal sea un espacio puramente ritual, con cuya creación algunos crean haber culminado ya las aspiraciones participativas de la población, mientras en otros casos pueden hacer propuestas operativas, que sean escuchadas y que obtengan respuesta pública por parte del gobierno municipal. De la misma manera, los jurados ciudadanos (o las propuestas de presupuestos) pueden tomarse como un mandato rotundo o como una de las muchas voces que se escuchan en un proceso político. En cualquier caso y aunque en muchas de las experiencias que analizaremos a continuación no haya una voluntad explícita de trascender las funciones meramente consultivas, su potencial impacto en las políticas locales podría ser uno de los argumentos para ir más allá de los Consejos consultivos.

Finalmente, el tercer gran aspecto que hemos señalado es la capacidad que pueden demostrar los procesos participativos de generar «mejores ciudadanos», desde el punto de vista de su interés y de su implicación con los temas colectivos. Los rendimientos sustantivos de los procesos participativos difícilmente pueden limitarse a la toma de decisiones concretas, olvidando su rol potencial como escuela de ciudadanía (AKKERMAN y otros, 1999; BARNES, 2000). Es decir, se trata de que los participantes, una vez terminada la experiencia, se muestren más predispuestos a volver a participar, con un mayor grado de confianza en las virtudes de la colaboración con los otros y con una mayor confianza en su propia capacidad de hacerse escuchar. Enlazando con una de las líneas de trabajo más populares de los últimos años podríamos decir que los mecanismos participativos jugarían un papel como instrumentos creadores de capital social ${ }^{8}$.

Este potencial educativo ha aparecido de forma más o menos explícita en el análisis de muy diversas experiencias participativas. Para el caso del

\footnotetext{
${ }^{8}$ Por tanto, nos situamos en aquella perspectiva que defiende la posibilidad de crear capital social estructuralmente inducido (MalONEY y otros, 1999) y en desacuerdo con la noción de Putnam (1993) que le atribuye unas raíces más históricas y un proceso de gestación interminablemente largo.
} 
CMBS, por ejemplo, una de sus principales virtudes es la capacidad que ha tenido de generar dinámicas de diálogo y de consenso entre los participantes, y de contribuir a generar una cultura del bienestar (SARASA y GuiU, 2001). Sin embargo, los efectos educativos de este tipo de instrumentos sólo llegan a una pequeña parte de la población, que precisamente por formar parte del tejido asociativo se puede considerar que ya está fuertemente politizada. El reto estará en los mecanismos de participación de base personal, y especialmente en aquellos que pretenden llegar no sólo a los ciudadanos interesados o implicados con los temas sino también, o sobre todo, a los ciudadanos con menos información. Experiencias como las de los jurados ciudadanos demuestran que gente que no está dispuesta a participar de forma continuada acepta hacerlo en mecanismos puntuales. La práctica participativa en este tipo de espacios les incrementa su respeto por las autoridades, les ayuda a comprender la complejidad de la vida colectiva, y puede contribuir a la educación de los participantes en los valores y las prácticas democráticas.

No obstante, también están claros los límites habituales de los instrumentos de participación en sus efectos educativos. Básicamente, la mayoría de estos instrumentos sólo llegan a un grupo reducido de ciudadanos, con lo cual los efectos educativos tienen dificultad para extenderse al conjunto de la población. Los Presupuestos Participativos son uno de los mecanismos que en alguna de las experiencias brasileñas han logrado un mayor porcentaje de participación, llegando sólo a un $1 \%$ de la población.

Dos condiciones serán importantes para superar este handicap. Primero, potenciar la visibilidad de los mecanismos participativos, difundiendo su realización más allá de los colectivos participantes a través de los medios de comunicación. Por ejemplo, los Presupuestos Participativos son conocidos por un $50 \%$ de la población, mientras que los resultados de los jurados ciudadanos o los consejos consultivos en este sentido son muy irregulares. Segundo, dar continuidad a los procesos participativos, de forma que estos efectos educadores puedan tener un carácter acumulativo a través de diversas experiencias. De este modo se podría enfrentar el otro gran problema detectado: el hecho de que tras una euforia inicial cuando se ha participado en un proceso interesante y que en principio ha generado una mayor voluntad de implicación colectiva, ésta se va diluyendo con el paso del tiempo ante la vuelta al bussiness as usual, es decir, a la realidad cotidiana de una vida política sin espacios previstos para escuchar la voz de los ciudadanos, que va convirtiendo aquella experiencia participativa en un recuerdo cada vez más lejano e irrelevante (BLANCO y otros, 2000). 
REAL-2002, núm. 290. FONT, JOAN y BLANCO, ISMAEL. PARTICIPACIÓN LOCAL. MÁS ALLÁ ...

REAL 290 (SEPTIEMBRE-DICIEMBRE, 2002)

En síntesis, aunque en muchos casos los consejos consultivos municipales hayan tenido una función muy positiva en sus respectivas localidades, acostumbran a presentar déficits en cuanto a representatividad, capacidad de influir en las políticas y de constituirse en amplias escuelas de ciudadanía. En el próximo apartado veremos hasta qué punto aquellos instrumentos de participación que han tratado de innovar respecto al funcionamiento habitual de dichos consejos consiguen superar estas limitaciones.

\section{3. ¿CÓMO IR MÁS ALLÁ DE LOS CONSEJOS CONSULTIVOS MUNICIPALES?}

El trabajo empírico que se describe a continuación proviene de un estudio realizado para la Escola d'Administració Pública de la Generalitat catalana, cuyo trabajo de campo se realizó durante la primavera del año 2000. En el mismo se incluyó un estudio exhaustivo de todo lo que se había hecho en este terreno en los municipios catalanes de más de 50.000 habitantes, así como una recogida selectiva de algunas experiencias especialmente significativas desarrolladas en municipios más pequeños ${ }^{9}$. En total, se trata de 50 experiencias de participación ciudadana, que a pesar de sus muy diversos formatos tienen precisamente en común el hecho de no ser Consejos consultivos con el formato tradicional ${ }^{10}$. En la mayor parte de los casos el análisis se basó principalmente en una entrevista a algún responsable local (técnico o político), así como en el análisis de la documentación existente, aunque para algunas experiencias se realizaron entrevistas complementarias a otros actores ${ }^{11}$. Empezaremos describiendo

\footnotetext{
${ }^{9}$ En Cataluña hay 19 municipios de más de 50.000 habitantes. De ellos provienen 41 de las 50 experiencias, mientras que las restantes proceden de otros 8 municipios de menor tamaño. Sólo 1 de los 19 municipios no había desarrollado ninguna experiencia de las aquí tratadas, mientras que en 2 de ellos no fue posible obtener respuesta. A pesar de la vocación de exhaustividad en estos 19 municipios sin duda dejamos de localizar algunos casos concretos, que resultaban especialmente complejos de encontrar en municipios grandes como Barcelona, donde no hay un único informante que conozca las actividades desarrolladas por los distintos departamentos.

${ }^{10}$ Se trataba siempre de incluir experiencias que hubieran tenido algún tipo de reconocimiento institucional y por tanto se excluían iniciativas ciudadanas que no hubieran tenido esta pretensión de formalización. Entendíamos por proceso de participación aquel en que los participantes y/o los impulsores del proceso habían tenido voluntad de que sirviera para influir directa o indirectamente en la elaboración de políticas públicas locales.

11 Sin duda el uso exclusivo de estas fuentes supone un riesgo de posibles sesgos, dado que algunos entrevistados mostraban una visión muy positiva y edulcorada de los procesos. Precisamente por ello hemos obviado el análisis de algunos aspectos en los que contábamos con menos información fiable y hemos profundizado más en las experiencias en las que hemos podido disponer de un abanico más amplio de información.
} 
REAL-2002, núm. 290. FONT, JOAN y BLANCO, ISMAEL. PARTICIPACIÓN LOCAL. MÁS ALLÁ ...

PARTICIPACIÓN LOCAL. MÁS ALLÁ DE LA DEMOCRACIA ASOCIATIVA

de forma panorámica los grandes tipos de experiencias que aparecen en la muestra, para pasar después a analizarlas en función de los tres criterios citados.

\subsection{Tipos de mecanismos}

Aunque cada una de las experiencias ha sido bautizada con criterios propios y atendiendo a las circunstancias locales, en bastantes casos podemos encontrar procesos que se inspiran en un número limitado de metodologías. Así, el mecanismo más abundante en la muestra son las Agendas 21 locales, que aparecen en 10 municipios. Esto se debe a la coincidencia entre el trabajo de campo y un momento de máxima expansión de estos procesos participativos (FonT y SUBIRATs, 2001), por lo que se hace difícil saber cuál puede ser su grado de consolidación y, por tanto, si volveríamos a encontrarnos una realidad de este tipo al repetir el estudio dentro de 10 años.

El segundo instrumento más habitual son los consejos consultivos con formato innovador. Es decir, aquellos que siguen en buena medida el modelo de los consejos consultivos municipales, pero introduciendo alguna modificación significativa en su funcionamiento, sea en los mecanismos de selección de los participantes, con presencia de ciudadanos a título individual, o en los colectivos a los que se pretende incorporar (niños). De hecho, podríamos dividir el grupo en función de este criterio, entre las experiencias más abundantes (Consejos Infantiles), que recibieron un fuerte impulso a mitad de los años 90 siguiendo el modelo italiano, respecto a aquellos consejos que incorporan ciudadanos elegidos al azar y que responden a un momento más reciente (Sant Feliu de Llobregat).

Aparecen 6 casos de Planes estratégicos. Como en el caso de les A21L, se trata de un proceso que puede adoptar mecanismos de funcionamiento diversos, como prueban los casos aquí analizados y que incluso puede tener un componente participativo muy intenso o muy débil. En cualquier caso se trata de un mecanismo en fase menos experimental, que muy probablemente seguirá siendo importante a medio plazo. También hay 6 casos de Jurados ciudadanos, aunque 2 de ellos se desarrollaran en el mismo municipio (Montornès). También en este caso se trata de un mecanismo innovador que llega a Cataluña a mediados de los años 90, a partir de varias experiencias desarrolladas en el País Vasco.

El último instrumento con presencia significativa recibe nombres muy diversos, pero responde a lo que podríamos llamar Mesas de barrio, que 
REAL-2002, núm. 290. FONT, JOAN y BLANCO, ISMAEL. PARTICIPACIÓN LOCAL. MÁS ALLÁ ...

REAL 290 (SEPTIEMBRE-DICIEMBRE, 2002)

tienen en común la voluntad de acercar el debate municipal a cada uno de los barrios del municipio o del distrito, con un formato que va más allá de la representación exclusiva de les entidades locales. Otros instrumentos participativos con una presencia menor ( 2 experiencias en cada caso) serían los referéndums, planes integrales, planes estratégicos sectoriales y presupuestos participativos.

En este conjunto de experiencias encontramos dos realidades significativamente distintas. En primer lugar tenemos lo que podemos considerar mecanismos de participación en sentido estricto, es decir, creados exclusivamente para vehicular la participación ciudadana, sea de forma puntual (Jurados ciudadanos) o permanente (Consejos infantiles). Pero casi la mitad de las experiencias aquí descritas son al mismo tiempo más y menos que mecanismos participativos, dado que se trata de procesos más amplios de debate y elaboración de políticas: los planes estratégicos, las Agendas 21 o el propio debate del Plan de Acción Municipal, no son mecanismos concretos sino procesos que integran, dándole más o menos importancia según el caso, dinámicas muy diversas de participación ciudadana.

Además, muchos de estos procesos integran metodologías participativas muy diversas. Así, para elaborar los presupuestos participativos de Sabadell se ha utilizado una estrategia de Investigación Acción Participativa (IAP), pero también la metodología EASW (European Awereness Scenario Workshops), de la misma manera que en algunas Agendas 21 o planes estratégicos se ha combinado la celebración de fórums con encuestas o páginas web interactivas. Por tanto, en las próximas páginas estaremos hablando de una realidad muy diversa, no sólo en sus detalles organizativos, sino en su propia lógica y razón de ser.

\subsection{Representatividad de los participantes}

En este apartado queremos analizar a los protagonistas de estos mecanismos participativos, es decir, a los colectivos a los que se pretende escuchar e implicar en el proceso. Quiénes son, cuál es el volumen de participantes o cuáles las similitudes y diferencias entre los participantes reales y el conjunto del colectivo al que se quería escuchar serán algunos de los temas que examinaremos. Empezaremos estableciendo una distinción muy básica en función de cuáles sean los colectivos a los que se pretende hacer participar: grupos organizados, ciudadanos a título individual o una combinación de ambos. Posteriormente analizaremos en detalle cada una de estas 3 posibilidades para ver cómo ha funcionado en la práctica la com- 
REAL-2002, núm. 290. FONT, JOAN y BLANCO, ISMAEL. PARTICIPACIÓN LOCAL. MÁS ALLÁ ...

PARTICIPACIÓN LOCAL. MÁS ALLÁ DE LA DEMOCRACIA ASOCIATIVA

binación de ciudadanos y grupos o cuál ha sido el grado de éxito a la hora de movilizar a la ciudadanía.

Los instrumentos que se dirigen de forma exclusiva a las asociaciones son minoritarios en este trabajo (6 casos). Obviamente ésta no es una realidad representativa del conjunto de los instrumentos de participación locales, sino resultado de la propia definición del objeto de estudio. Por tanto, los mecanismos que aquí aparecen como formados sólo por asociaciones son experiencias excepcionales que huyen del formato del consejo consultivo. Entre las restantes, encontramos un número significativo de experiencias de ambos tipos: de composición mixta (asociaciones y ciudadanos, 26 casos), y sólo con ciudadanos a título individual (15).

La diversidad de modelos de funcionamiento es mucho mayor al fijarnos en los instrumentos con participación mixta (cuadro 1). La fórmula más rígida es también la menos empleada, es decir, aquella que atribuye una cuota determinada a ciudadanos y otra a grupos. Esta opción permite introducir la dosis deseada de cada colectivo y evitar la dominación de alguno de ellos, pero al mismo tiempo obliga a fijar unos criterios difíciles a priori para los que no existen claras argumentaciones. Así, mientras en los Consejos territoriales de Sant Feliu se ha optado por la coexistencia de ambos colectivos en condiciones de paridad, en el Consejo de 100 jóvenes de Barcelona hay un amplio predominio de jóvenes a título individual, en lo que supone de facto la existencia de una bicefalia en los órganos de representación de los jóvenes barceloneses: a título individual en este organismo y a través de las asociaciones en el Consejo local de la Juventud.

En los casos restantes se opta por no establecer ningún número concreto, lo que implica acceso libre de unos y otros. Aunque ello pudiera parecer que va a generar mucha mayor presencia de personas a título individual el efecto es exactamente el opuesto: la combinación de falta de información, de interés o de experiencia lleva en la práctica en estos casos a una rotunda mayoría asociativa. Esto se refuerza a menudo por el uso de distintos mecanismos movilizadores, pues mientras a las asociaciones se las invita por medio de una carta a cada una de ellas, se confía en que la participación de los restantes ciudadanos se producirá como resultado de la presencia de algunos carteles o anuncios en la prensa local. Así, los participantes a título individual fueron una pequeña minoría de alrededor del 5\% del total en experiencias como el Plan estratégico de Viladecans o la Agenda 21 de Manresa. En el caso de que esta combinación de sectores se produzca en mecanismos que prevén una participación continuada en el tiempo también tiende a producirse una presencia más continua del sector asociativo, mientras que la asistencia individual es más puntual, con las 
REAL-2002, núm. 290. FONT, JOAN y BLANCO, ISMAEL. PARTICIPACIÓN LOCAL. MÁS ALLÁ ...

REAL 290 (SEPTIEMBRE-DICIEMBRE, 2002)

consecuencias que ello implica tanto en la información como en la capacidad de influencia en las decisiones.

Cuadro 1. Tipología de mecanismos con participación mixta, algunos ejemplos (número de casos)

\begin{tabular}{lll}
\hline $\begin{array}{l}\text { Proporción fija de } \\
\text { participantes } \\
(15)\end{array}$ & $\begin{array}{l}\text { Participación abierta } \\
(4)\end{array}$ & $\begin{array}{l}\text { Participación abierta, } \\
\text { con vías } \\
\text { complementarias (5) }\end{array}$ \\
\hline $\begin{array}{l}\text { Consells teritorials de } \\
\text { Sant Feliu } 12\end{array}$ & Agenda 21 (casi todas) & Pla estratègic de Girona \\
$\begin{array}{l}\text { Jurat ciutadà de } \\
\text { Montornès-I }\end{array}$ & $\begin{array}{l}\text { Projecte educatiu de } \\
\text { ciutat (Reus) }\end{array}$ & $\begin{array}{l}\text { Pla integral de Joventut } \\
\text { (Mataró) }\end{array}$ \\
\hline
\end{tabular}

Existen dos variantes a este patrón mayoritario, que tratan de potenciar las voces de los ciudadanos individuales. Una de ellas es la extensión a los mismos (o a parte de ellos) de los mecanismos de invitación personalizada. Otra, la puesta en marcha de mecanismos paralelos de consulta a este sector, en forma de encuestas, páginas web, línea telefónica para sugerencias o de otros mecanismos puntuales como las postales utilizadas por el plan estratégico de juventud de Mataró. Esta diversidad de mecanismos provoca también resultados muy dispares en la capacidad de movilización final. Cuando se trata de participación activa y en persona muchas de las actividades relativamente exitosas se sitúan entre las 100 y las 200 personas, aunque existan casos excepcionales como las 700 personas de la Agenda 21 de Manlleu o del Plan estratégico de Rubí. Las cifras crecen si incorporamos formas de intervención menos costosas como las encuestas, con las que se llega a menudo a cifras de 800-1000 participantes.

Finalmente, también entre los mecanismos que solo prevén la participación de ciudadanos a título personal existe una gran diversidad de criterios respecto a como seleccionar los participantes (cuadro 2). La primera gran diferencia separa los mecanismos que no restringen la participación y aquellos que sí lo hacen. Entre los primeros podemos distinguir entre los que tienen una clara vocación de participación universal (referéndums) y aquellos con carácter abierto, pero que dejarían de ser viables si realmente decidieran participar buena parte de los sectores a los que se pretende consultar. Entre éstos, el grado de movilización real es muy diverso y puede ir desde las pocas personas hasta superar las 300 en el primer fórum ciudadano de

12 Mantenemos el nombre original sin traducción cuando hacemos referencia a una experiencia concreta. 
REAL-2002, núm. 290. FONT, JOAN y BLANCO, ISMAEL. PARTICIPACIÓN LOCAL. MÁS ALLÁ ...

PARTICIPACIÓN LOCAL. MÁS ALLÁ DE LA DEMOCRACIA ASOCIATIVA

Sant Boi. Si en ninguno de estos mecanismos «abiertos» se ha llegado nunca a movilizar al $1 \%$ de su audiencia potencial, en las dos convocatorias de referéndum de la muestra la participación llegó al 56\% del censo en Palamós y al 38\% en Sant Andreu. Además de contar con una participación más limitada, en los mecanismos «abiertos» a menudo se produce una participación que, aunque se haga a título individual, está protagonizada de forma casi exclusiva por miembros de asociaciones.

Cuadro 2. Mecanismos con participación individual: ¿quién participa?

\begin{tabular}{lllll}
\hline Todos & Abierto & $\begin{array}{l}\text { Representantes } \\
\text { electos }\end{array}$ & $\begin{array}{l}\text { Representantes } \\
\text { aleatorios }\end{array}$ & Designados \\
\hline $\begin{array}{l}\text { Referèndums } \\
(2)\end{array}$ & $\begin{array}{l}\text { Forums } \\
\text { ciudadanos } \\
\text { (3) }\end{array}$ & $\begin{array}{l}\text { Consejos } \\
\text { infantiles (4) }\end{array}$ & $\begin{array}{l}\text { Jurados } \\
\text { ciudadanos } \\
(5)\end{array}$ & $\begin{array}{l}\text { Café amb } \\
\text { l'alcalde }\end{array}$ \\
\hline
\end{tabular}

En los otros mecanismos, el número de participantes está limitado y por tanto es necesario establecer criterios para decidir quienes serán éstos. Los tres procedimientos utilizados han sido los mismos que han funcionado a lo largo de la historia para elegir representantes de la población: la designación por las autoridades, el sorteo y la elección. En la primera categoría encontramos únicamente la experiencia del Café con el alcalde de Reus, en el que en cada sesión se elige a los representantes ciudadanos que se consideran más apropiados en función del tema a debatir. La selección aleatoria es el mecanismo utilizado en las experiencias de Jurados ciudadanos, buscando así cierta representatividad sociológica de los participantes. Es decir, convertir el reducido número de participantes (de 48 a 93) en una reproducción a escala reducida de la composición social de cada municipio. Este criterio se ha utilizado también en mecanismos ya aludidos como el Consejo de 100 jóvenes de Barcelona o en las encuestas de opinión que se han hecho en el marco de algunos planes estratégicos. Finalmente existen los mecanismos donde los representantes han sido elegidos, como en algunos de los Consejos infantiles, aunque en bastantes casos esta elección haya combinado criterios democráticos con otros de carácter meritocrático.

¿Qué relación hay entre dos objetivos deseables como extensión de la participación y representatividad de los participantes? ¿Presentan contradicciones de manera que si maximizamos uno sacrificamos el otro o por el contrario, se refuerzan mutuamente? Los datos procedentes de estas experiencias más bien apuntan hacia la falta de relación entre ambas dimensiones, pero en cambio a la existencia de relación entre ambos aspectos y 
la metodología utilizada (cuadro 3). Así, si los referéndums son capaces de generar una participación considerable es probable que provoquen también un resultado bastante representativo, mientras que en los Jurados ciudadanos, si funciona bien el proceso de reclutamiento, se producirá un resultado representativo, aunque generalmente con una participación poco numerosa. Lo que sí parece claro es que los mecanismos con participación abierta pueden dar lugar a más participantes que otros, pero al mismo tiempo tenderá a ser menos representativa del conjunto de la población.

Cuadro 3. Representatividad y número de participantes (mecanismos con participación exclusivamente individual)

\begin{tabular}{llll}
\hline \multicolumn{3}{c}{ Número de participantes } \\
\hline Representatividad & Pocos & Algunos & Muchos \\
\hline Baja & Café amb l'alcalde & Plens Arbúcies & \\
& Consell infants- & Forums Sant Boi & \\
& Viladecans & & \\
\hline Media & Consell Infants (2) & Referèndums \\
\hline Alta & 5 Jurados ciutadans & \\
& Consell Infants Reus & \\
\hline
\end{tabular}

En síntesis, el debate sobre qué voces deben ser escuchadas ha sido resuelta de formas extremadamente diversas, en algunos casos a partir del convencimiento que la fórmula adoptada es la más apropiada y en otros como resultado de la negociación con otros actores locales. La tendencia a dar un protagonismo que tradicionalmente no habían tenido a los ciudadanos no organizado es clara, pero a la vez, el rol de las asociaciones ha seguido siendo importante en el grupo mayoritario de experiencias que combinaba la participación individual con la de carácter asociativo, apareciendo tensiones tanto en la búsqueda de la fórmula apropiada para combinar ambos tipos de participación, como en la voluntad de maximizar al mismo tiempo el número y la representatividad de los participantes.

\subsection{El impacto en las políticas}

El cuadro 4 pretende clasificar el grado y tipo de influencia en las políticas de los instrumentos analizados aquí. Por un lado, establecemos una gradación respecto al grado de influencia, aunque no disponemos de ningún indicador empírico sólido y nos basamos en las declaraciones de los entrevistados. Por otro lado, utilizamos la distinción entre aquellos instrumentos que de forma prioritaria pretenden contribuir a definir prioridades 
REAL-2002, núm. 290. FONT, JOAN y BLANCO, ISMAEL. PARTICIPACIÓN LOCAL. MÁS ALLÁ ...

PARTICIPACIÓN LOCAL. MÁS ALLÁ DE LA DEMOCRACIA ASOCIATIVA

e identificar problemas, respecto a aquellos que quieren intervenir en las decisiones más concretas de políticas a adoptar.

Cuadro 4. Grado y tipo de influencia en las políticas. Algunos ejemplos (número casos)

\begin{tabular}{llll}
\hline & Baja & Media & Alta \\
\hline Definición & $\begin{array}{l}\text { Cafè amb l'alcalde } \\
\text { Jurat ciutadà } \\
\text { de St Quirze (4) }\end{array}$ & $\begin{array}{l}\text { Agenda 21 de Rubí } \\
\text { Presupostos } \\
\text { participatius de } \\
\text { Sabadell (8) }\end{array}$ & $\begin{array}{l}\text { Programa } \\
\text { Urban de Santa } \\
\text { Coloma } \\
\end{array}$ \\
& & $\begin{array}{l}\text { Pla Integral de } \\
\text { Joventut a } \\
\text { Mataró (3) }\end{array}$ \\
\hline Decisión & 3 Consells d'Infants & Consell de 100 joves & 2 Referèndums \\
& $\begin{array}{l}\text { Consells territorials } \\
\text { de Sant Feliu (4) }\end{array}$ & Jurat ciutadà de & Corbera (5) \\
& & & $\begin{array}{l}\text { ciutadans de } \\
\text { Montornès (4) }\end{array}$ \\
& & & \\
\hline
\end{tabular}

¿Hay mecanismos con más capacidad de influencia que otros? Sí aparecen tendencias claras en algunos casos, aunque hay una gran diversidad de situaciones para un mismo mecanismo, apuntando a que la voluntad política es tan o más decisiva que el tipo de instrumento utilizado. El caso más claro y heterogéneo es el de los Jurados ciudadanos. Mientras que en dos municipios el cambio de equipo de gobierno ha llevado a descartar las conclusiones del proceso, en Corbera hay posibles signos de cumplimiento parcial y en Montornés ya se está implementando las resoluciones del primer consejo, a pesar de que éstas fueran contrarias a la voluntad del equipo de gobierno. Un caso menos extremo pero donde también se aprecia cierta diversidad sería el de los consejos consultivos innovadores. Así, hay indicios de mayor capacidad de influencia del Consejo de 100 jóvenes de Barcelona (ampliación del horario nocturno del Metro, nueva línea de autobús nocturno) que de los Consejos de Sant Feliu, y lo mismo ocurre con los Consejos infantiles, que han tenido algún impacto en un par de municipios, pero a los que se concede un rol puramente ritual o educativo en los restantes.

En otros mecanismos, más que hablar de impacto desigual en las políticas, encontramos una situación en la que las resoluciones son tan amplias o generalistas que se hace difícil analizar su grado de cumplimiento real. Esto ocurre en algunas Agendas 21, pero también en los presupuestos participativos de Sabadell, que han señalado un amplio listado de necesidades con escasa concreción y priorización, de manera que comprometen muy poco al equipo de gobierno. Por el contrario, aunque sea difícil hacer afir- 
REAL-2002, núm. 290. FONT, JOAN y BLANCO, ISMAEL. PARTICIPACIÓN LOCAL. MÁS ALLÁ ...

REAL 290 (SEPTIEMBRE-DICIEMBRE, 2002)

maciones contundentes al basarnos sólo en 2 casos, parece haber un mecanismo al que su amplia visibilidad pública y la nitidez de las alternativas discutidas da un mandato difícil de contradecir: los referéndums. En ambos casos, aunque el carácter legal de la consulta no fuera vinculante, ningún actor local ha defendido que los resultados no debían ser respetados.

En síntesis, el formato participativo no es el único elemento determinante para que un proceso sea más o menos influyente a la hora de la verdad. Un mecanismo que produce unos efectos casi vinculantes en el caso brasileño como los presupuestos participativos ha provocado un impacto marginal en el único caso similar aquí analizado. También hemos observado resultados muy diversos de un mismo mecanismo en función del grado de apoyo con que éste contara por parte de distintos actores locales. Sin embargo, el formato sí cuenta. Una resolución más clara favorece que ésta pueda ser públicamente defendida con mayor facilidad, del mismo modo que la mayor visibilidad del proceso da también más fuerza moral a sus conclusiones.

\subsection{Los efectos educativos}

En muchas de estas experiencias de participación sus impulsores insisten en señalar que su objetivo principal era menos el de incorporar realmente la participación al proceso de toma de decisiones colectivas, que el de contribuir a crear unos espacios de diálogo y creación de ciudadanía, que contribuyeran al aprendizaje democrático de los participantes y a la creación de capital social en el municipio. ¿Existen indicios de que se haya hecho algún progreso en esta dirección?

En la mayor parte de experiencias podemos hablar de una respuesta positiva, por mucho que los efectos sean limitados. Si empezamos por aquellos casos en que parece claro que este efecto no se ha producido podemos detectar un doble tipo de situaciones: o bien experiencias con un número muy limitado de participantes que ya se conocían y donde por tanto este mecanismo concreto no ha supuesto ningún avance significativo desde este punto de vista, o bien actuaciones muy puntuales y que por tanto no tenían siquiera pretensiones de dejar ningún tipo de legado cultural. En la mayoría de los casos restantes sí se apunta a algún tipo de efectos, aunque éstos sean de tipos muy diversos. Así, las jornadas de Badalona contribuyeron a crear un mayor grado de coordinación entre las asociaciones participantes y el proceso de elaboración del Plan estratégico de Rubí contribuyó decisivamente a la formación de la Federación de Aso- 
REAL-2002, núm. 290. FONT, JOAN y BLANCO, ISMAEL. PARTICIPACIÓN LOCAL. MÁS ALLÁ ...

PARTICIPACIÓN LOCAL. MÁS ALLÁ DE LA DEMOCRACIA ASOCIATIVA

ciaciones de Vecinos y de la Agrupación local de comerciantes. Este mismo proceso de mejora del clima político se ha producido en Mataró a partir del Plan estratégico sobre juventud, pero en este caso entre las fuerzas políticas locales, que pasaron a tratar este tema desde una perspectiva de mayor confrontación a un escenario de diálogo y colaboración.

En otros casos, además del posible aprendizaje democrático se ha producido una sensibilización de los participantes hacia los temas tratados, como en caso de algunas Agendas 21 o del Plan para la Sociedad de la Información de Terrasa. Pero quizás los casos más interesantes son aquellos donde la visibilidad del proceso ha permitido trascender las fronteras de los participantes y llegar a otros sectores ciudadanos. Este proceso se ha dado a través de llevar los debates de los Consejos infantiles a las escuelas en algunos de los municipios implicados, de provocar un debate interno en las asociaciones a partir del debate público del PAM en Manresa o incluso de la difusión mediática de los Cafés con el alcalde de Reus y las expectativas que éstos han generado.

Respecto a la relación entre tipo de instrumento y efectos educativos nos encontramos en este caso con efectos muy débiles entre ambas cosas. El entorno político que ha rodeado al proceso participativo se configura como la variable predominante, por encima de la metodología concreta adoptada. Así, por ejemplo, el referéndum de Palamós, con un mayor grado de implicación de los actores locales, tiene una capacidad mucho mayor de creación de capital social que una experiencia mucho más dirigida en exclusiva desde el Ayuntamiento como en el caso de Sant Andreu. En el caso de los Consejos infantiles se observa cómo el factor clave es la relación de éstos con el exterior, es decir, hasta qué punto los representantes infantiles realizan su función de intermediación y explican el proceso en los centros educativos que los han elegido. Finalmente, los Jurados ciudadanos serían un ejemplo de mecanismo con efectos relativamente homogéneos, con una moderada capacidad de suscitar un creciente interés por los temas locales entre los participantes, pero a menudo con poca capacidad de crear complicidad con el movimiento asociativo, que los percibe como mecanismos donde pierden su protagonismo.

En síntesis, los mecanismos participativos han provocado todo un conjunto de consecuencias positivas en la red de relaciones entre ciudadanos, asociaciones y gobierno local, afectando a las relaciones en el interior o entre varias de estas categorías. Además, los tipos de efectos tienen cierta relación con los instrumentos adoptados, pero éstos muestran una gran diversidad de resultados. Las características concretas de su aplicación, así como el entorno en que fueron aplicados y la reacción de apoyo u oposi- 
REAL-2002, núm. 290. FONT, JOAN y BLANCO, ISMAEL. PARTICIPACIÓN LOCAL. MÁS ALLÁ ...

REAL 290 (SEPTIEMBRE-DICIEMBRE, 2002)

ción que generaron se configuran en este caso como variables decisivas para entender su potencial efecto educativo.

\section{CONCLUSIONES}

En este trabajo hemos analizado todo un conjunto de nuevos mecanismos de participación ciudadana desarrollados a lo largo de los últimos años. Su rasgo distintivo fundamental se producía en el propio sujeto llamado a participar, en la medida en que en la mayor parte de los casos se iba más allá del proceso habitual de dar voz a los grupos organizados, para tratar de incorporar también a la ciudadanía a título individual. Todo ello en el marco de muy diversas fórmulas metodológicas, algunas creadas fundamentalmente como mecanismos participativos y otras desarrolladas como procesos más amplios de planificación estratégica que incorporaban espacios participativos en mayores o menores dosis.

Pero ¿han sido estos nuevos mecanismos de alguna utilidad?, ¿han servido para superar las limitaciones de las fórmulas participativas tradicionales? Para tratar de dar respuestas a estas preguntas hemos empezado tratando de señalar cuáles eran las principales limitaciones habituales de los Consejos consultivos y las hemos centrado en tres grandes ámbitos: la representatividad, el escaso impacto en el proceso de elaboración de políticas públicas y la capacidad de educación democrática de los mecanismos. Es posible que la lista no sea exhaustiva, que la gravedad de estos problemas sea muy diversa según el caso concreto o la perspectiva de cada actor, pero parece difícilmente objetable que éstos son tres terrenos en los cuales es posible ir más allá de los resultados cotidianos de muchos consejos consultivos. Sin duda, esto no significa una descalificación global de la labor realizada por estos consejos que ha sido extremadamente diversa, que aún ha estado muy poco estudiada en profundidad y que, en muchos casos, ha generado rendimientos indiscutibles.

Hemos tratado de analizar la superación de estos déficit en otros mecanismos de participación local, a partir de un estudio de cincuenta experiencias catalanas, procedentes en su mayor parte de municipios grandes o medianos. El tema de la representatividad ha tratado de abordarse desde fórmulas muy diversas. En algunos casos se producía un cambio total de estrategia y se daba todo el protagonismo a los ciudadanos a título individual, en otras se les daba un rol en un espacio común con el movimiento asociativo y en otras se creaban espacios paralelos o compartimentados para dar cabida a ambas realidades. La apuesta a favor de dar voz a los ciu- 
REAL-2002, núm. 290. FONT, JOAN y BLANCO, ISMAEL. PARTICIPACIÓN LOCAL. MÁS ALLÁ ...

PARTICIPACIÓN LOCAL. MÁS ALLÁ DE LA DEMOCRACIA ASOCIATIVA

dadanos constituye un importante paso adelante respecto a la capacidad de representación que tienen las asociaciones en los consejos municipales. Sin embargo, la práctica analizada apunta resultados muy desiguales y en los que no resulta posible maximizar todos los objetivos deseables. Así, en algunos instrumentos la convivencia teórica de ciudadanos y grupos se convierte en la práctica en una situación claramente hegemónica y favorable a los segundos, en otras se apuesta por una participación generalizada (referéndums) o aleatoria (Jurados ciudadanos) que permite escuchar de forma representativa pero puntual la voz de los ciudadanos no organizados, sin que los intentos de combinar de forma permanente la voz de ciudadanos y asociaciones hayan conseguido funcionar bien. Finalmente, las fórmulas que apuntan a crear espacios paralelos para ambos grupos abren el gran interrogante de cómo pueden integrarse a posteriori los resultados de ambas vías.

Por lo que respecta a la influencia en las políticas, estas experiencias dan lugar a resultados desiguales y que a veces resulta aún prematuro evaluar. En cualquier caso, y aunque no pueda hablarse de codecisión generalizada ni dispongamos de indicadores claros para hacer un juicio global, sí parece que en bastantes de estos procesos se ha ido más allá de lo que es habitual en la mayor parte de Consejos consultivos, en cuanto a capacidad de influencia en las políticas, sea en su proceso de elaboración o en aspectos más concretos vinculados a su implementación. Procesos participativos con resultados claros y concretos y con mayor visibilidad pública tendrán mucha más capacidad de incidencia real en las políticas que aquellos que den lugar a un amplio catálogo de recomendaciones generalistas.

En el terreno de la capacidad de educación democrática algunos de estos instrumentos poseen dos grandes ventajas respecto a la dinámica de la mayoría de los consejos consultivos. En primer lugar, abren la puerta a un número mayor de participantes, muchos de los cuales disponen habitualmente de menores oportunidades participativas, por lo que el público susceptible de realizar el proceso de aprendizaje democrático está mucho más presente. En segundo lugar, unas reglas de funcionamiento donde las distancias jerárquicas son menores y donde se favorece un diálogo horizontal entre los propios participantes y a veces con los mismos políticos crea también un espacio más abonado para dicho aprendizaje.

Aunque, como hemos visto, buena parte de las metodologías citadas se han aplicado con mucha flexibilidad, de manera que encontramos una gran diversidad en su implementación, se observa que cada una de ellas contribuye en mayor medida a superar unos u otros problemas. Así, un proceso largo y continuado puede ser más favorable a un proceso de aprendizaje 
que contribuya a crear capital social (plan estratégico), pero al mismo tiempo puede favorecer que el carácter decisional del proceso se diluya y resulte menos visible que en una experiencia con un calendario muy limitado (referéndum, jurado ciudadano). De la misma manera, un énfasis muy grande en la representatividad nos puede llevar a valorar fórmulas de tipo aleatorio, que tengan como consecuencia que el impacto educativo quede limitado a un número muy reducido de participantes. En cualquier caso, este conjunto de experiencias sí parece apuntar a que poner en marcha mecanismos participativos que vayan más allá de una participación estrictamente asociativa puede contribuir a superar algunos de los límites de los Consejos consultivos, especialmente de aquellos que más rotundamente han denunciado el movimiento asociativo.

\section{Referencias}

Abers, Rebbeca (1997) «Inventing local democracy. Neighbourhood organising and participatory policy-making in Porto-Alegre, Brazil», Tesis doctoral, Los Angeles, University of California.

AKKerman, Tiskje et al. (1999): «Intercative policy-making as deliberative democracy? Learning from new policy-making practices in Amsterdam», Comunicación presentada en el Congreso de APSA, Washington.

Barnes, M. (2000) «Researching Public Participation» en Pratchett, L (ed.) Renewing local democracy? The modernisation agenda in British local government, Londres, Frank Cass.

Blanco, Ismael; GimÉnez, Laura; y PinedA, Laia (2001): «Estudi d'evaluació dels Consells ciutadans: una flor no fa estiu» en SÀnchez, J. (coord.). Participació ciutadana: govern local.- Els Consells ciutadans, Editorial Mediterránea.

Brugué, Quim et al. (2001): «Consejos consultivos en Barcelona: un balance», en Font, J. (ed.) Ciudadanos y Decisiones Públicas, Barcelona, Ariel.

CoHen \& Rogers (1995): Associative Democracy, Verso.

Delap, Clare (2001): «Jurados ciudadanos en el Reino unido», en FonT (ed.): obra citada.

Fishrin, James (1995) Democracia y deliberación, Barcelona, Ariel. 
Font, Joan y Blanco, Ismael (2001): «Conclusiones» en Font, J. (ed.) op. cit.

Font, Núria y SubiRATS, Joan (2001): Local y sostenible, Icaria.

MaLONEY et al. (2000): «Social capital and urban governance: adding a more contextualized 'tod-down' perspectice», Political Studies, 48.

Navarro, Clemente J. (1999): El sesgo participativo, Madrid, CSIC.

Putnam, Robert (1993): Making democracy work, Princeton University Pres.

Sarasa y Guiu (2001): «El Consejo Municipal de bienestar social de Barcelona», en FonT (ed.): obra citada.

Schlozman, Verba \& Brady (1999): «Participation's not a paradox», British Journal of Political Science, 25.

SubIRATS, Joan (2001): «Nuevos mecanismos participativos y democracia: promesas y amenazas» en FonT, J. (ed.) op. cit.

Subirats, Joan; Blanco, Ismael; Brugué, Quim; Font, Joan; GomÀ, Ricard; Jarque, Marina; Medina, Lucía (2001): Experiències de participació ciutadana en els municipis catalans. Barcelona, Escola d'Administració Pública. 


\section{Reseñas de legislación y jurisprudencia}

